\section{Long-term impact of subclinical postpartum thyroiditis}

A small proportion of women develop transient autoimmune thyroid dysfunction (hyperthyroidism, hypothyroidism, or both) within 12 months of childbirth. Although it has been shown that a significant number of women with overt disease will go on to develop permanent hypothyroidism, information is lacking on the long-term clinical picture for those who present with subclinical disease.

Of an initial intake of 213 women with hypothyroidism diagnosed 3-10 months postpartum, 172 returned for follow-up in this single-center Iranian study. The women underwent 12-24 months of treatment with levothyroxine and were then followed up for a median of $16 \pm 19$ months (until hypothyroidism recurred or the study ended). Of those with subclinical disease before treatment $(n=27), 59 \%$ developed either subclinical $(44 \%)$ or overt (15\%) hypothyroidism after withdrawal of treatment. Disease recurred after treatment withdrawal in approximately two-thirds of women who had overt disease before treatment, with $36 \%$ again developing overt disease. Time to recurrence was longer, and post-treatment serum levels of thyroidstimulating hormone were lower, in women with subclinical hypothyroidism than in those with overt disease.

This is the largest study of its kind to date and, although limited by selection bias and loss of patients to follow-up, it demonstrates that subclinical postpartum hypothyroidism can lead to permanent thyroid failure, which suggests that prompt diagnosis and longterm follow-up are of particular importance for affected women.

Rebecca Doherty

Original article Azizi F (2005) The occurrence of permanent thyroid failure in patients with subclinical postpartum thyroiditis. Eur J Endocrinol 153:367-371

\section{Leptin levels predict features of the metabolic syndrome}

Several studies have linked obesity with the metabolic syndrome. Obesity, however, is not a prerequisite for the metabolic syndrome and little is known about the underlying mechanisms and pathways that relate to development of this syndrome. Franks and colleagues, therefore, tested the hypothesis that leptin is associated with the development of features of the metabolic syndrome, independently of obesity.

Franks and colleagues used data from a prospective population-based cohort, which consisted of 748 adults. Data included participants' height, weight and blood pressure, insulin, glucose and lipid levels, and plasma leptin levels, collected at baseline and at 5 and 10 years' follow-up. At the end of the last available follow-up, there were 105 incident cases of the metabolic syndrome.

Baseline leptin levels were significantly associated with development of obesity. After adjusting for BMI, baseline leptin levels significantly predicted the development of insulin resistance and glucose intolerance. Leptin levels, however, did not significantly predict changes in blood pressure or lipidemia. More importantly, leptin levels significantly predicted the development of the metabolic syndrome, after controlling for obesity, in a dose-dependent manner.

The authors conclude that leptin levels predict the development of the metabolic syndrome and this is specifically related to glucose intolerance and insulin resistance. This association is independent of baseline obesity. Further studies are, however, required to confirm the independence of these associations.

Marie Lofthouse

Original article Franks PW et al. (2005) Leptin predicts a worsening of the features of the metabolic syndrome independently of obesity. Obes Res 13: 1476-1484

\section{ED-71: a promising new candidate for the treatment of osteoporosis?}

Treatment with a new active vitamin D, ED-71, effectively and safely increased lumbar and hip bone mineral density (BMD) in osteoporotic patients who also received vitamin $\mathrm{D}_{3}$ supplementation, according to the findings of a randomized, double-blind, placebo-controlled trial.

In all, 219 osteoporotic patients took part in the trial, and were randomly assigned to placebo or $0.50,0.75$ or $1.00 \mu \mathrm{g} /$ day ED-71 for 12 months, in addition to 200 or 400 IU/day vitamin $D_{3}$. Lumbar and hip BMD showed 